\title{
LUT
}

University

\section{Optimized Morphological Analysis in Decision-Making}

Buzuku Shqipe, Kraslawski Andrzej

This is a Author's accepted manuscript (AAM) version of a publication

published by Palgrave Macmillan, Cham

in Advances in Systematic Creativity

DOI: 10.1007/978-3-319-78075-7_14

Copyright of the original publication: (c) The Author(s) 2019

Please cite the publication as follows:

Buzuku S., Kraslawski A. (2019) Optimized Morphological Analysis in Decision-Making. In:

Chechurin L., Collan M. (eds) Advances in Systematic Creativity. Palgrave Macmillan, Cham

This is a parallel published version of an original publication.

This version can differ from the original published article. 


\title{
Optimized Morphological Analysis in Decision-Making
}

\author{
Shqipe Buzuku ${ }^{1 *}$ and Andrzej Kraslawski ${ }^{1,2}$
}

\begin{abstract}
Morphological analysis is one of the methods most widely used in identification, formulation, and structuring complex problems with the aim of seeking optimal solutions. When extended with cross consistency assessment, morphological analysis becomes an iterative method. The aim of this chapter is to propose a modeling approach for using extended morphological analysis and to complement it with sensitivity analysis for optimization and time reduction over the iteration process. First, the aim is to recognize the connection between systems engineering requirements and project management activities, while incorporating multiple design dimensions and categories. Second, the chapter extends the application of the creative design approach. The target is to recognize and analyze the conflicts between the design activities, and aims at creating alternative options and solutions to resolve conflicts.
\end{abstract}

Keywords: morphological matrix, sensitivity analysis, optimization, decision-making, project management

\section{Introduction}

Creativity has emerged as a driving force to innovation processes and systems' design for the industry of current and future generations. Many assessment methods for innovation are already developed and can be identified in the literature. One of these methods is the Theory of Inventive and Problem Solving, also known by its Russian acronym TRIZ, was developed by Altshuller (1984), and is a remarkable instrument to boost creativity in engineering. 
TRIZ focuses on creative idea generation process in company's environment (Chechurin 2016). Another promising method widely used to support systematic creativity is morphological analysis (MA), developed by Zwicky (1969). MA is presented as a promising method for generation of new concepts and finding the best solution to support decision-making. Both TRIZ and MA are well-known and commonly used methods for complex problem solving, including techniques for idea generation and divergent thinking.

MA is a non-quantified modelling method for identifying, structuring and studying complex problems. It is a suitable tool for addressing highly complex and ill-structured problems, also known as "wicked problems", which are becoming increasingly frequent in a globalized world. The term wicked problems introduced by Rittel and Webber (1973) is used to describe complex social problems that "do not have an enumerable set of potential solutions" and are multidimensional and non-quantifiable, with inherent technical, social, institutional, and political difficulties of addressing them (Ney 2012). Such problems are found for instance in strategy development, product innovation, policy design, etc. Hence, MA is used to structure and investigate the behavior of wicked problems and discover solutions (Ritchey 2011).

Moreover, wicked problems may deal with long-term policy design, planning and complexity management, and involve many individuals and organizations with overlapping roles. Tackling wicked problems in decision-making for industrial mega-project management can happen, e.g., while seeking optimization of time, cost and quality, and reduction or management of risk. This type of problem is usually formulated with many contradictory requirements, and involves many system's elements from various functional areas. In such cases, the application of traditional multi-objective decision-making approaches has limitations. Hence, this situation allows applying a morphological approach developed by Zwicky (1969) and Ritchey (2011). Moreover, today's industry is constantly under high pressure to generate competitive advantages and improve their resource-efficiency. For example, time reduction plays is a 
significant target in overall industrial project management. To address this problem, several methods and tools exist focused for optimization.

Therefore, MA its proposed to be further improved by integrating with sensitivity analysis in this chapter, which has never been done before. Sensitivity analysis (SA) is an important task in multi-criteria decision-making. Moreover, SA deals with the investigation of potential changes and errors in variables and assumptions, and their impacts on the results of underlying models. SA, applied post-hoc to decision models, deals with uncertainties related to the decision outcomes and/or to the preferential judgements (i.e. value function and criterion weights). The objective is to find out how the options' ranking changes by any modification made on the decision models (Mysiak 2010). Applying SA shows great potential of time reduction over the morphological analysis iterative part of process.

In order to elaborate in the topic, the document is organized as follows: Background and literature review of morphological analysis and sensitivity analysis is discussed in Section 2. The proposed solution for optimization of MA through SA is explained in Section 3. To test the theory there is a numerical illustration to demonstrate the proposed method in Section 4 , as well as the results, managerial implications and recommendations. In the Section 5 the research conclusions, limitations and future work are presented.

\section{Methodological background}

This section explains the overall process of the study by providing a brief explanation of each method used. An innovative approach of applying MA complemented with SA to this problem is explored with the aim of seeking optimal solutions. Moreover, the methodological foundation of the study is based on the iterative nature of the cross-consistency assessment (an integral part of MA) as an opportunity for further optimizing the overall process of MA. A decision tree is generated based on the morphological space and cross-consistency analysis is conducted in order to present the benefits of the method. 


\subsection{Morphological analysis}

Morphological analysis was originally introduced by Zwicky (1969), as a non-quantified modelling method for identifying, structuring and studying complex problem. In principle, MA is based on the "divide and conquer" technique, which tackles a problem using two basic approaches: "analysis" and "synthesis" - the basic method for developing scientific mode (Ritchey 2018). This technique is a decomposition method that breaks down a system into subsystems with several attributes and selects the most valuable alternative (Yoon and Park 2005). In other words, MA systematically categorizes the possible combinations of subsystems. The strength of the technique lies in its ability to provide structured models for complex problems into simpler problems, rather than offering solutions (Pidd 2009). MA can be considered as a contemporary heuristic method that has clear links with traditional approaches in different fields of problem solving (Arciszewski 2018). It has been subject of academic research over many years and it has been applied to a wide variety of fields and contexts such as shown in Table 14.1.

Table 14.1. Literature survey of morphological analysis applications

\begin{tabular}{lll}
\hline Application & References \\
\hline Engineering and product & (Jimenez and Mavris 2010), (Ostertagová et al. 2012), \\
design & (Ölvander, Lundén, and Gavel 2009), (Sholeh, Ghasemi, \\
& and Shahbazi 2018)
\end{tabular}

Design theory and (Chen and Lai 2010), (Ritchey 2011), (Zeiler 2018), Architecture (Kannengiesser, Williams, and Gero 2013)

Future studies and Scenario (Ritchey 2011), (Lopes Correia da Silva 2011), (Duczynski development 2017), (Johansen 2018), (Voros 2018), (Haydo 2018) Technological (Yoon 2008), (Feng and Fuhai 2012), (Takane et al. 2009), Forecasting/Technology (Yoon, Park, and Coh 2014) 
Foresight

Management science, Policy

(Ajith and Ganesh 2009), (Pidd 2009), (Plauché et al. analysis and 2010), (Kuriakose et al. 2010), (Storbacka and Nenonen

Organizational design, 2012), (Im and Cho 2013), (Seidenstricker, Scheuerle, and Strategic planning

Linder 2014), (Buzuku and Kraslawski 2015), (Buzuku, Kraslawski, and Harmaa 2015), (Teles and Freire de Sousa 2017), (Teles and Freire de Sousa 2018), (Duczynski 2018)

Security safety and Design (Louise, Mapule, and le Roux 2009)

studies

Creativity, Innovation and (Seidenstricker and Linder 2014), (Frow et al. 2015), Knowledge Management (Geum, Jeon, and Lee 2016), (Jeong et al. 2016)

Modeling Theory, OR (Ritchey and de Waal 2007), (Plauché et al. 2010), (Ritchey methods and GMA 2012), (Williams and Bowden 2013), (Ritchey 2014), (Ritchey 2018), (Ritchey 2018)

MA has been widely used for identifying possible variable combinations in different disciplines. It enables problem representations using a number of dimensions, which are permitted to assume the number of conditions (Eriksson and Ritchey 2002). The identified conditions in each dimension can be combined to derive all the possible alternatives that can solve the problem. MA requires a multidisciplinary group of 5-7 experienced experts (Yoon and Park 2005), representing different aspects of the issues involved. In particular, MA provides a strong possibility for identifying unexpected combinations of new concepts. The basic procedure of the morphological analysis consists of the following stages:

- Problem definition,

- Analysis,

- Synthesis, 
- Exploration of results

\subsubsection{Problem definition}

In this stage, the problem definition is to determine suitable problem characteristics. The individual problem solver or a facilitated group brainstorms to define problem characteristics, also referred to as parameters. In the specific context of the study, the problem is to establish a systematic integrative approach for problem solving, in such a way that the integrated approach supports complex problem solving for efficient industrial management.

The specific goal is to utilize the MA and optimization of cross-consistency assessment (CCA) through sensitivity analysis as modeling methods supporting decision-making in case of wicket problems such as strategy development, product innovation or policy design.

\subsubsection{Analysis}

After problem definition, in the analysis stage the main task in MA is generation of a morphological field, which consists of identification and specification of the essential parameters or variables of the problem and generation of the design alternatives or values for each parameter or component (i.e., a morphological class). At this stage, creative and design thinking is desirable to generate a set of potential solutions as large as possible. Finally, the morphological box (field) is created with a large solution space (see Figure 14.1).

\begin{tabular}{|c|c|c|c|c|}
\hline Parameter A & Parameter B & Parameter C & Parameter D & Parameter E \\
\hline Value A1 & Value B1 & Value C1 & Value D1 & Value E1 \\
\hline Value A2 & Value B2 & Value C2 & Value D2 & Value E2 \\
\hline Value A3 & $\ldots$ & Value C3 & $\ldots$ & Value E3 \\
\hline Value A4 & & $\ldots$ & & $\ldots$ \\
\hline$\cdots$ & & & & \\
\hline
\end{tabular}


Figure 14.1 A morphological matrix consisting of five parameters and their ranges of values. The matrix represents $144(=4 \times 2 \times 3 \times 2 \times 3)$ formal configurations such as $(A 2, B 1, C 2$, D2, E1).

\subsubsection{Synthesis}

The synthesis stage presents the CCA proposed by Ritchey (2015). CCA involves evaluation of the feasibility of all combinations of all values and of all the variables. CCA assesses compatibility for each value via pairwise comparison (the comparison of a value from one column, or morphological class, against another value from another morphological class). The purpose of CCA matrix is to filter out infeasible configurations. The CCA is represented in a multidimensional matrix, which then is reduced to find an approximation for an optimal solution. The process is iterative in nature and time-consuming. Manual configuration evaluations in physical settings are based on a cross consistency matrix. Therefore, optimization of the iteration process is a relevant thing to do.

\subsubsection{Exploration}

In this stage, the internally consistent combinations are synthesized, with the objective to reduce the total set of possible combinations. This step is termed assessment of consistent configurations. The cross-consistency assessment (CCA) is computed to ensure the consistency of pairwise comparisons. This step is based upon the insight that there may be numerous mutually incompatible pairs of conditions in the morphological matrix. Zwicky calls MA "totally research" which it enables in an unbiased way attempts to derive all the possible solutions of any given problem.

The main advantage of the MA method is visualization support. With the usage of CCA, MA facilitates the calculation of a large amount subset of consistent configurations, which is impossible to do manually. It may help to discover new relationship or configurations, which may not be so evident, or which might have been overlooked by other unstructured or less- 
structured methods. Importantly, it encourages the identification and investigation of boundary conditions, i.e. the limits and extremes of different contexts and factors. In addition, the MA method provides various kinds of representations of the consistent configuration space. Such representations help groups to structure discussions and support decision-making.

\subsection{Sensitivity analysis}

Sensitivity analysis serves a wide range of applications. The SA can be applied in:

(i) Decision-making for identifying critical value/criterion, testing robustness, and riskiness of decision;

(ii) Communication for increasing credibility and confidence and

(iii) Modelling process, for better understanding of input-output relationship and for understating the model needs and restrictions (Mysiak 2010).

SA deals with the investigation of potential changes and errors associated to the inputs and assumptions, and their impact on the results of underlying models. While the impact of uncertainties on the decision outcomes is mostly analyzed by statistical modelling and simulation, the preferential judgments are object of uncertainty during the modelling of weights and value function (Mysiak 2010). In normal mathematical modeling and simulation, SA is used to evaluate how the changes of the variables or assumptions affect the results. When analyzing a CCA matrix, all values contained within it (result of the evaluation and weighting of values by a panel of experts) can be considered as inputs and each of these inputs has a specific impact in the solution space (the output) generated by the evaluation of the matrix. The use of SA helps highlighting the combinations with higher impact within the solution space and of a specific value.

\section{Proposed solution}


In this section, an innovative solution is proposed and presented with a case example. One method capable of complementing and optimizing MA is SA. According to Ferretti, Saltelli, and Tarantola (2016), sensitivity analysis has been applied in a very diverse range of fields, typically to calculate and estimate uncertainty of models. However, to the best of our knowledge, it has never before been applied with MA. Furthermore, the proposed use of SA aims to find the most influential combinations of the cross-consistency assessment matrix to reduce the time of iteration. In this study, MA is integrated with SA as a concept with high potential for time reduction.

\subsection{Optimization of morphological analysis through sensitivity analysis}

Morphological analysis is an approach successfully used for solving the problems where many solutions and alternatives are possible (Zwicky 1969). It consists in analysis of all possible combinations of input variables and next identification of the most promising solution. The exhaustive list of permutations of the values then forms the complete morphological space (often referred as Zwicky box by Ritchey (2018). The method makes complications arise, however, due to the fact that it is not always feasible to manually test all (or even many) of the potential solutions identified within a morphological space (Eriksson and Ritchey 2002). Therefore, Ritchey (2011) proposed the computer implementation of this method. A binary or numerical CCA matrix, generated through a panel of experts' evaluation over the combinatory interactions of the parameters and their value pairs generates a set of solution space (Ritchey 2015). The process is iterative in nature and it should be repeated as many times as required, to achieve a manageable solution space. A manageable solution space should have enough combinations of solutions for decision makers to have a clear pathway, but not too many, so decision-making is complex within the solution space again (Ritchey 2018). On the other hand, SA has been used in a multitude of fields (Ferretti, Saltelli, and Tarantola 2016), often aimed to test the uncertainty levels derived of variable assumptions in mathematical models. 
However, the presented approach proposes yet a different use of SA as a concept. The suggested approach proposes the use of SA to evaluate the effect of the change of each value in the matrix in the overall set of combinations of a specific value. In other words, if a pairwise value is changed from value "A" to "B" (zero to one in a binary matrix, or different numerical values in a numerical matrix), it will decrease the overall amount of combinations of value " $A$ " in the solution space. Since every iteration of value revaluation takes time to the panel of experts, knowing which cells affect the most to the overall number of combinations of certain value can save a significant amount of time for revaluation a large CCA matrix.

\section{Numerical illustration}

To test the theory, a cross-consistency assessment (CCA) matrix with values from 1 to 3 and dimensions as shown in Figure 14.2 was created in a loop of randomly generated values for one thousand iterations in MatLab, and the relative amount of combinations in each iteration is calculated according to Equation 1:

$$
R W x_{(a, b)}=\frac{K x_{(a, b)}}{\sum_{i=1}^{R} \Sigma_{j=1}^{C} K x_{(i, j)}}
$$

Equation 1 shows the method used to calculate the relative weight (RW) of an element pair, in respect to the total combinations " $K$ ", which are unique combinations of value " $\mathrm{x}$ ", for the position ( $a, b)$ (where " $a$ " is the row and " $b$ " is the column in the CCA matrix) calculated as indicated by Ritchey (2010).

The term " $\mathrm{R}$ " denotes the total of rows;

"C" denotes the total of columns, and

"ij" and "j" are the counters to calculate the total of every cell. 


\begin{tabular}{|c|c|c|c|c|c|c|c|c|c|c|c|c|c|c|c|c|c|c|c|}
\hline & P2,V1 & $\mathbf{P 2}, \mathbf{V} 2$ & P2,V3 & P2,V4 & P2,V5 & P3,V1 & P3,V2 & P3,V3 & P3,V4 & P3,V5 & P4,V1 & P4,V2 & P4,V3 & P5,V1 & P5,V2 & P5, V3 & P5, V4 & P5,V5 & P5,V6 \\
\hline P1,V1 & 3 & 1 & 2 & 3 & 1 & 3 & 1 & 1 & 2 & 3 & 1 & 3 & 3 & 3 & 2 & 3 & 3 & 3 & 3 \\
\hline P1,V2 & 1 & 3 & 1 & 2 & 1 & 1 & 3 & 3 & 2 & 1 & 1 & 1 & 3 & 1 & 3 & 3 & 1 & 1 & 3 \\
\hline P1,V3 & 3 & 3 & 3 & 2 & 1 & 3 & 3 & 3 & 1 & 1 & 3 & 3 & 3 & 1 & 3 & 3 & 1 & 1 & 3 \\
\hline P1,V4 & 1 & 2 & 3 & 3 & 3 & 2 & 1 & 2 & 3 & 3 & 1 & 3 & 3 & 3 & 2 & 3 & 3 & 3 & 3 \\
\hline P1,V5 & 1 & 2 & 1 & 1 & 3 & 3 & 1 & 1 & 3 & 3 & 1 & 2 & 2 & 1 & 3 & 1 & 1 & 1 & 1 \\
\hline P1,V6 & 1 & 2 & 1 & 1 & 3 & 1 & 1 & 1 & 3 & 3 & 1 & 2 & 2 & 3 & 1 & 1 & 1 & 1 & 3 \\
\hline P2,V1 & & & & & & 3 & 1 & 1 & 2 & 2 & 1 & 1 & 3 & 3 & 2 & 3 & 1 & 3 & 3 \\
\hline P2,V2 & & & & & & 1 & 3 & 3 & 2 & 2 & 1 & 2 & 3 & 2 & 2 & 3 & 1 & 1 & 3 \\
\hline P2,V3 & & & & & & 3 & 1 & 1 & 1 & 3 & 3 & 3 & 3 & 3 & 1 & 3 & 1 & 1 & 1 \\
\hline P2,V4 & & & & & & 2 & 2 & 3 & 1 & 2 & 2 & 2 & 2 & 3 & 2 & 3 & 1 & 2 & 3 \\
\hline P2,V5 & & & & & & 1 & 1 & 1 & 3 & 3 & 2 & 3 & 3 & 3 & 1 & 1 & 3 & 3 & 3 \\
\hline P3,V1 & & & & & & & & & & & 3 & 3 & 3 & 3 & 3 & 3 & 1 & 1 & 1 \\
\hline P3,V2 & & & & & & & & & & & 3 & 2 & 1 & 1 & 3 & 3 & 1 & 1 & 1 \\
\hline P3,V3 & & & & & & & & & & & 1 & 2 & 3 & 1 & 1 & 3 & 1 & 1 & 1 \\
\hline P3,V4 & & & & & & & & & & & 1 & 3 & 3 & 3 & 2 & 2 & 2 & 2 & 3 \\
\hline P3,V5 & & & & & & & & & & & 1 & 3 & 3 & 3 & 2 & 2 & 2 & 2 & 3 \\
\hline P4,V1 & & & & & & & & & & & & & & 2 & 1 & 2 & 3 & 2 & 1 \\
\hline P4,V2 & & & & & & & & & & & & & & 2 & 2 & 2 & 3 & 3 & 2 \\
\hline P4,V3 & & & & & & & & & & & & & & 3 & 3 & 3 & 2 & 2 & 3 \\
\hline
\end{tabular}

Figure 14.2 Example cross-consistency assessment (CCA) matrix revaluation layout highlighting the cells of value " 3 " and marking in red the cells generating $75 \%$ of the total combinations of value " 3 ".

Figures 14.3 and 14.4 show the premise behind the CCA plus SA concept. The value pair P1V3 and P2V1 generates a set of 27 unique combinations of value "3" (Figure 14.3), while the total unique combinations of value " 3 " for the row of P1V3 is 81 . In contrast the value pair P1V2 and P2V2 (Figure 14.4) produces only 6 unique combinations of value "3". When all value pairs are evaluated, a total of 993 unique combinations of value "3" are produced from the entire matrix. Therefore, the cell P1V3, P2V1 hosts $2.7 \%$ of the total unique combinations of value "3", while the cell P1V2, P2V2 generates only $0.6 \%$. Figure 14.2 shows an example of the benefits of conducting sensitivity analysis into the CCA matrix. The picture highlights all cells with value " 3 " (a total of 108). Considering a scenario, where it is desirable to reduce the overall amount of combinations of value " 3 ", the panel of experts in charge of evaluating the iteration may intuitively review only the combinations of value "3" (108 highlighted in Figure 14.2, both red and yellow) saving about $56 \%$ of time required to evaluate the whole CCA matrix. Furthermore, after applying SA it is possible, in the example case, to focus on the cells with higher amount of overall unique combinations of value " 3 ". Highlighted in red are the cells of value " 3 " that host more than $1 \%$ of the overall unique combinations of value " 3 ". By 
revaluating only the cells highlighted in red it is necessary to iterate only 31 (highlighted in red) out of the 108 cells of value pairs marked with a " 3 ". In this manner, (assuming that the revaluation of each value takes the same amount of time) it is possible to save, in the example, $71 \%$ of the time of iteration, when only the cells of value " 3 " are evaluated, or $84 \%$ of the time of re-evaluating all the 247 values in the CCA matrix. Furthermore, by evaluating the value of only the cells highlighted in red it is possible to reduce up to $75 \%$ of the total amount of unique combinations of value " 3 " from the example given. 


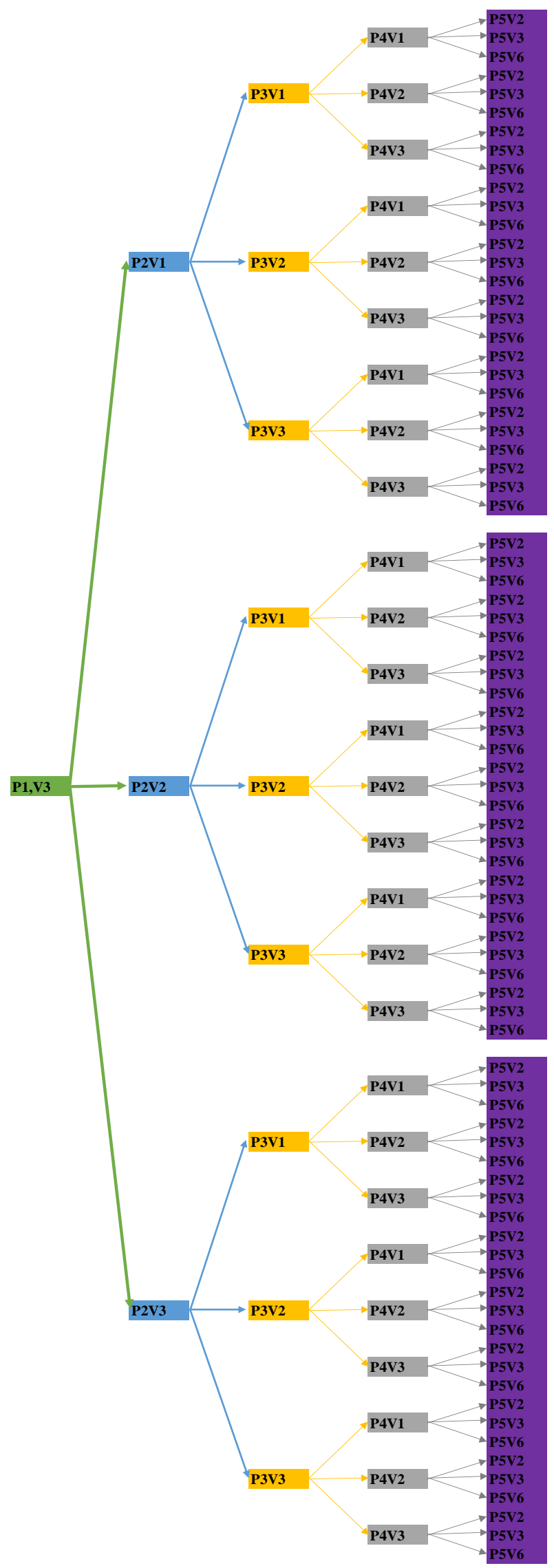

Figure 14.3 Example of a large morphological decision tree for a value pair of high amount of unique combinations of value " 3 ". 


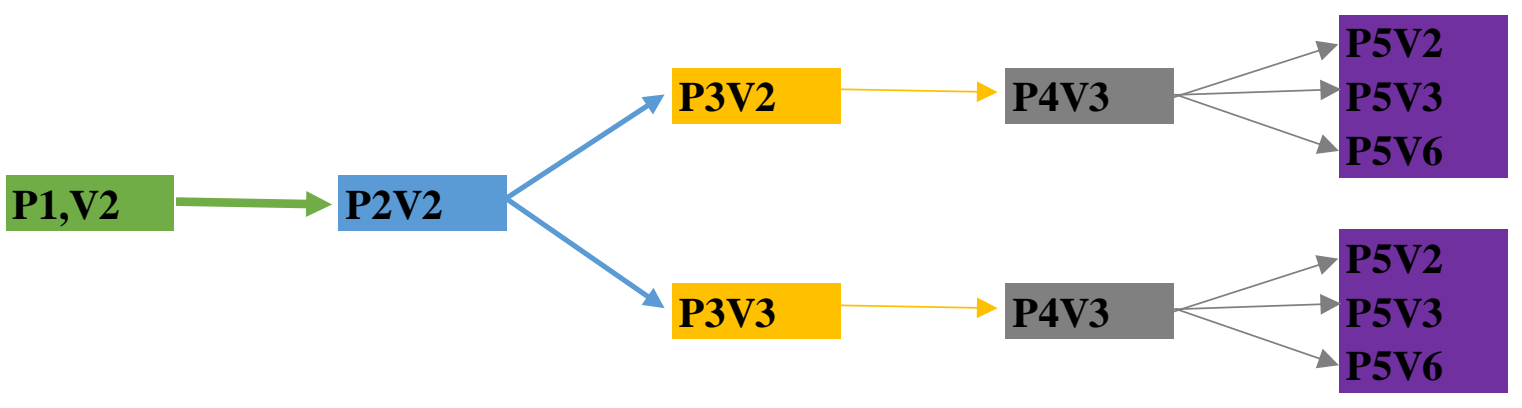

Figure 14.4 Example of a small morphological decision tree for a value pair of low amount of unique combinations of value " 3 ".

\subsection{Results}

Using a multi-paradigm numerical computing software, a one thousand iterations loop was created to generate and evaluate through SA a cross-consistency assessment matrix of random values from " 1 " to " 3 " and of the dimensions shown in Figure 14.2. In addition, a sensitivity limit of $1 \%$ was set, meaning that every cell of the CCA matrix containing more than $1 \%$ of the total amount of combinations of a certain value is then highlighted as target for revaluation when needed. From the analysis of the results, it was found that, over the one thousand iterations loop, it is possible to reduce the iteration time in average $63.1 \%$ when only the combinations of a certain value are evaluated, with a maximum of $88.8 \%$ and minimum of $24.7 \%$. When compared to the evaluation of all cells in the matrix, the average time saving was $87.8 \%$ with a maximum of $96.4 \%$ and a minimum of $77.7 \%$. Furthermore, the relative amount of combinations contained within the SA highlighted cells averaged $73.9 \%$, while the maximum registered was $91.8 \%$ and the minimum $53.1 \%$.

In other words, over the one thousand loop iterations test, an average of $73.9 \%$ of the combinations of any value from " 1 " to " 3 " can be reduced by evaluating only $36.9 \%$ of the cells of the CCA matrix with a specific value, or $12.2 \%$ of all the cells of the whole CCA matrix, hence saving $63.1 \%$ and $87.8 \%$ of the time respectively. The considered timesaving assume an equal time required to evaluate every cell in the CCA matrix. The concept of optimization of 
MA through SA, as presented in this section, holds a great potential for time reduction of the MA iterative part of the process.

\subsection{Managerial implications}

The results of this research reveal important implications for decision makers and managers. Several managerial suggestions are from results analysis. Decision makers are able to define which combinations within their industrial case require more attention and which combinations will be out of the box with less significance.

The proposed approach of integrating MA with SA as a concept with high potential for time reduction, can effectively and efficiently be used for managing industrial complexity. The major contribution of the research is to facilitate decision-making process in industry. The optimization of CCA based model helps to facilitate decision makers and managers to determine the relative importance of the combination sets in the CCA.

The results of the method approach could encourage managers to implement the integrated approach of MA with SA with high effect on time reduction over the iteration process. In addition, SA results help managers determine the influence of the experts' opinion, by evaluating the inputs against their impact.

\subsection{Recommendations}

The sensitivity limit should be chosen accordingly to the size of the CCA matrix. For a CCA matrix of e.g. 80 values, a sensitivity limit of $1 \%$ would be too low, and result in the majority of cells with the desired value being highlighted (and required to iterate), thus mostly neglecting the advantage of SA application. On the other hand, a $1 \%$ sensitivity limit into a CCA matrix of e.g. 500 values may exclude too many cells, therefore reducing the reach of combinations reduction by applying SA. As a general rule of thumb, the authors recommend a sensitivity 
limit of $\sim(1 / n) x 2$, where " $n$ " stands for the number of cells of the CCA matrix. In the example case, $(1 / 247) \times 2=0.8 \% \sim 1 \%$. SA is advised to be used in order to reduce the total amount of combinations of the desired value only for a few iterations, but not to the length of generating the final solution. After the solution space has been significantly reduced, it is recommended that the experts evaluate a slightly extended pool of solutions. This is under the reasoning that the most optimal solution is not necessarily contained within a segregated group of optimal values, but may sometimes include compromises in some combinations.

\section{Conclusions and future work}

MA is a widely used method for decision making in planning and management, and it is often complemented with other methods to tackle its limitations. Nevertheless, the authors are unaware of research in the existing literature on optimization of MA and CCA through SA and focus on time reduction in management of complex projects.

This paper proposed a model for optimization of morphological analysis and cross-consistency assessment through SA to support decision-making in project management. The integration of MA and SA, under the test conditions presented, has shown promising potential for time reduction. MA optimization was achieved by reducing iteration time. The two morphology constituents, i.e. dimensions and values, show different characteristics. The construction of dimensions requires a significant level of expert judgments compared to that of a value, since the combination of dimensions should thoroughly reflect type of interaction. To succeed in developing new innovative concepts, dimensions should be mutually exclusive and collectively exhaustive. Furthermore, the iterative process of MA and cross-consistency assessment receives a significant improvement using SA.

SA as an optimization tool can be performed with any set of values, in every iteration, and target any specific value ("1", "2" or " 3 " from the example, but not limited to those of the 
example). It is a powerful and adaptable tool capable of obtaining desirable solutions in a fraction of the time required otherwise. The analysis can also be automated in a spreadsheet. In addition, the sensitivity limits can be tailored to adapt the method to CCA matrixes of any dimensions, adding another layer of flexibility. This work has some identified limitations. It is worthy to mention that the test conditions are randomly generated values and the time reduction is considering equal time requirements for evaluation of every value. Extensive application in real world cases may show different distributions. Nevertheless, the integration of the methods should, in any case and to some extent, optimize the iteration and revaluation process with significant time reductions. Moreover, it could be argued that when focusing only on a highlighted set of cells due to their value, the possibility exist that a good or optimal solution contained within the non-highlighted cells could be missed. Nevertheless, by narrowing the combinations to iterate, a good solution (if not the optimal) could be reached much faster, while in a complete iteration an optimal solution could still be lost among the many combinations to be reiterated. Furthermore, in order to prove or disprove this possibility, further research and application to real life case studies is required. In a real-world situation such as that studied here, MA has proved to be a successful approach for resolving complex problems and it can be applied with multidisciplinary group decision makers. Future research can directly include integration of risk and uncertainty assessment in all modifications of MA decisions.

\section{Acknowledgements}

This research was supported in part by The Research Foundation of Lappeenranta University of Technology [LUT Tukisäätiö grant number 122/16] and The Foundation for Economic Education, [Grant number 160039].

\section{References}


Ajith, Kumar J., and L. S. Ganesh. 2009. "Research on Knowledge Transfer in Organizations: A Morphology." Journal of Knowledge Management 13 (4):161-74. https://doi.org/10.1108/13673270910971905.

Altshuller, Genrikh. 1984. Creativity As an Exact Science. Pocket Mathematical Library. CRC Press.

Arciszewski, Tomasz. 2018. "Morphological Analysis in Inventive Engineering."

Technological Forecasting and Social Change 126:92-101.

https://doi.org/10.1016/j.techfore.2017.10.013.

Buzuku, Shqipe, and Andrzej Kraslawski. 2015. "Engineering Geology and Health and Safety. Application of Morphological Analysis to Policy Formulation for Wastewater Treatment." Notes of the Mining Institute 214:102-8.

Buzuku, Shqipe, Andrzej Kraslawski, and Kari Harmaa. 2015. "Supplementing Morphological Analysis with a Design Structure Matrix for Policy Formulation in a Wastewater Treatment Plant.” In Modeling and Managing Complex Systems, 9-18. Fort Worth (Texas, USA), 4-6 November 2015. https://doi.org/https://doi.org/10.3139/9783446447264.002.

Chechurin, Leonid. 2016. "TRIZ in Science. Reviewing Indexed Publications." Procedia CIRP 39:156-65. https://doi.org/10.1016/j.procir.2016.01.182.

Chen, Jocelyn Hua-Chu, and Cheng-Fang Lai. 2010. “The Theory of Morphological Analysis Applied to Western Apparel-a Case Study of Renaissance Era." International Journal of Computer Science and Network Security 10 (4):176-84.

Duczynski, Guy. 2017. "Morphological Analysis as an Aid to Organisational Design and Transformation.” Futures 86:36-43. https://doi.org/10.1016/j.futures.2016.08.001. 
2018. "Investigating Traffic Congestion: Targeting Technological and Social Interdependencies through General Morphological Analysis.” Technological Forecasting and Social Change 126 (February 2017):161-67. https://doi.org/10.1016/j.techfore.2017.05.019.

Eriksson, Tomas, and Tom Ritchey. 2002. "Scenario Development Using Computerised Morphological Analysis." Winchester International OR Conference.

Feng, Xu, and Leng Fuhai. 2012. "Patent Text Mining and Informetric-Based Patent Technology Morphological Analysis: An Empirical Study.” Technology Analysis and Strategic Management 24 (5):467-79. https://doi.org/10.1080/09537325.2012.674669.

Ferretti, Federico, Andrea Saltelli, and Stefano Tarantola. 2016. "Trends in Sensitivity Analysis Practice in the Last Decade." Science of The Total Environment 568:666-70. https://doi.org/10.1016/j.scitotenv.2016.02.133.

Frow, Pennie, Suvi Nenonen, Adrian Payne, and Kaj Storbacka. 2015. "Managing CoCreation Design: A Strategic Approach to Innovation.” British Journal of Management 26 (3):463-83. https://doi.org/10.1111/1467-8551.12087.

Geum, Youngjung, Hongseok Jeon, and Hakyeon Lee. 2016. "Developing New Smart Services Using Integrated Morphological Analysis: Integration of the Market-Pull and Technology-Push Approach.” Service Business 10 (3):531-55. https://doi.org/10.1007/s11628-015-0281-2.

Haydo, Peter A. 2018. "From Morphological Analysis to Optimizing Complex Industrial Operation Scenarios.” Technological Forecasting and Social Change 126:147-60. https://doi.org/10.1016/j.techfore.2017.06.009.

Im, Kwanyoung, and Hyunbo Cho. 2013. "A Systematic Approach for Developing a New 
Business Model Using Morphological Analysis and Integrated Fuzzy Approach." Expert Systems with Applications 40 (11):4463-77. https://doi.org/10.1016/j.eswa.2013.01.042.

Jeong, Seonkoo, Yujin Jeong, Keeeun Lee, Sungjoo Lee, and Byungun Yoon. 2016.

“Technology-Based New Service Idea Generation for Smart Spaces: Application of 5G Mobile Communication Technology." Sustainability 8 (11):1211. https://doi.org/10.3390/su8111211.

Jimenez, Hernando, and Dimitri Mavris. 2010. “An Evolution of Morphological Analysis Applications in Systems Engineering.” In 48th AIAA Aerospace Sciences Meeting Including the New Horizons Forum and Aerospace Exposition, 1-10. https://doi.org/doi:10.2514/6.2010-972.

Johansen, Iver. 2018. "Scenario Modelling with Morphological Analysis.” Technological Forecasting and Social Change 126 (February 2017):116-25.

https://doi.org/10.1016/j.techfore.2017.05.016.

Kannengiesser, Udo, Christopher Williams, and John Gero. 2013. "What Do the Concept Generation Techniques of Triz, Morphological Analysis and Brainstorming Have in Common." 19th International Conference on Engineering Design, ICED 20137 DS7507 (October 2015):297-300.

Kuriakose, Kocheril K., Baldev Raj, Satya S. A. V. Murty, and Pitchai I. Swaminathan. 2010. “Knowledge Management Maturity Models - A Morphological Analysis.” Journal of Knowledge Management Practice 11 (3):1-9.

Lopes Correia da Silva, Lydia. 2011. "Morphological Analysis of Electric Vehicles Introduction in Urban Traffic in Sao Paulo.” Future Studies Research Journal 3 (1):1437. 
Louise, Leenen, Modise Mapule, and Herman le Roux. 2009. “A Model for Peace Support Operations: An Overview of the ICT and Interoperability Requirements.” In Proceedings of the 4th International Conference on Information Warfare and Security, $1-10$.

Mysiak, Jaroslav. 2010. “Decision Methods.” 2010. http://www.netsymod.eu/mdss/mDSS_DECMETH.pdf.

Ney, Steven. 2012. Resolving Messy Policy Problems: Handling Conflict in Environmental, Transport, Health and Ageing Policy. The Earthscan Science in Society Series. Taylor \& Francis.

Ostertagová, Eva, Jozef Kováč, Oskar Ostertag, and Peter Malega. 2012. “Application of Morphological Analysis in the Design of Production Systems.” Procedia Engineering 48:507-12. https://doi.org/10.1016/j.proeng.2012.09.546.

Pidd, Michael. 2009. Tools for Thinking: Modelling in Management Science. Wiley.

Plauché, Madelaine, Alta de Waal, Aditi Sharma Grover, and Tebogo Gumede. 2010. "Morphological Analysis: A Method for Selecting ICT Applications in South African Government Service Delivery." Information Technologies \& International Development $6(1): 1-20$.

Ritchey, Tom. 2010. Wicked Problems, Social Messes: Decision Support Modelling with Morphological Analysis. Ritchey Consulting.

- 2011. Wicked Problems - Social Messes Decision Support Modelling with Morphological Analysis. Risk, Governance and Society. Springer Berlin Heidelberg. https://doi.org/10.1007/978-3-642-19653-9.

_ 2012. "Outline for a Morphology of Modelling Methods: Contribution to a General 
Theory of Modelling.” Acta Morphologica Generalis AMG Vol 1 (1):1-20.

. 2014. "Four Models about Decision Support Modeling." Acta Morphologica

Generalis AMG 3 (1):1-15.

_.2015. "Principles of Cross-Consistency Assessment in General Morphological Modelling." Acta Morphologica Generalis 4 (2):1-20.

—. 2018. "General Morphological Analysis as a Basic Scientific Modelling Method." Technological Forecasting and Social Change 126 (June 2017):81-91.

https://doi.org/10.1016/j.techfore.2017.05.027.

Ritchey, Tom, and Alex de Waal. 2007. "Combining Morphological Analysis and Bayesian Networks for Strategic Decision Support.” ORiON 23 (2):105-21. https://doi.org/10.5784/23-2-51.

Rittel, Horst W. J., and Melvin M. Webber. 1973. "Dilemmas in a General Theory of Planning.” Policy Sciences 4 (2):155-69. https://doi.org/10.1007/BF01405730.

Seidenstricker, Sven, and Christian Linder. 2014. “A Morphological Analysis-Based Creativity Approach to Identify and Develop Ideas for BMI: A Case Study of a HighTech Manufacturing Company.” International Journal of Entrepreneurship \& Innovation Management 18 (5/6):409-24. https://doi.org/10.1504/IJEIM.2014.064716.

Seidenstricker, Sven, Stefan Scheuerle, and Christian Linder. 2014. “Business Model Prototyping - Using the Morphological Analysis to Develop New Business Models.” Procedia - Social and Behavioral Sciences 148:102-9. https://doi.org/10.1016/j.sbspro.2014.07.023.

Sholeh, Mehdi, Ahmadreza Ghasemi, and Meisam Shahbazi. 2018. “A New Systematic Approach in New Product Development through an Integration of General 
Morphological Analysis and IPA.” Decision Science Letters 7:181-96.

https://doi.org/10.5267/j.ds1.2017.5.004.

Storbacka, Kaj, and Suvi Nenonen. 2012. "Competitive Arena Mapping: Market Innovation Using Morphological Analysis in Business Markets.” Journal of Business-to-Business Marketing 19 (3):183-215. https://doi.org/10.1080/1051712X.2012.638464.

Takane, Yoshio, Sunho Jung, Yuriko Oshima-Takane, Roger E. Millsap, and Alberto Maydeu-Olivares. 2009. "Multidimensional Scaling” 9 (3):219-42.

Teles, Maria de Fátima, and Jorge Freire de Sousa. 2017. “A General Morphological Analysis to Support Strategic Management Decisions in Public Transport Companies.” Transportation Research Procedia 22:509-18. https://doi.org/10.1016/j.trpro.2017.03.069.

—. 2018. "Linking Fields with GMA: Sustainability, Companies, People and Operational Research.” Technological Forecasting and Social Change 126:138-46. https://doi.org/10.1016/j.techfore.2017.05.012.

Voros, Joseph. 2018. “On a Morphology of Contact Scenario Space.” Technological Forecasting and Social Change 126:126-37. https://doi.org/10.1016/j.techfore.2017.05.007.

Williams, Peter B., and Fred D.J. Bowden. 2013. “Dynamic Morphological Exploration.” In 22nd National Conference of the Australian Society for Operations Research, 1-6.

Yoon, Byungun. 2008. “On the Development of a Technology Intelligence Tool for Identifying Technology Opportunity.” Expert Systems with Applications 35 (1-2):12435. https://doi.org/10.1016/j.eswa.2007.06.022.

Yoon, Byungun, Inchae Park, and Byoung-youl Coh. 2014. "Exploring Technological 
Opportunities by Linking Technology and Products: Application of Morphology Analysis and Text Mining." Technological Forecasting and Social Change 86:287-303. https://doi.org/10.1016/j.techfore.2013.10.013.

Yoon, Byungun, and Yongtae Park. 2005. “A Systematic Approach for Identifying Technology Opportunities: Keyword-Based Morphology Analysis.” Technological Forecasting and Social Change 72 (2):145-60.

https://doi.org/10.1016/j.techfore.2004.08.011.

Zeiler, Wim. 2018. “Morphology in Conceptual Building Design.” Technological Forecasting and Social Change 126:102-15. https://doi.org/10.1016/j.techfore.2017.06.012.

Zwicky, Fritz. 1969. Discovery, Invention, Research through the Morphological Approach. Macmillan.

Ölvander, Johan, Björn Lundén, and Hampus Gavel. 2009. “A Computerized Optimization Framework for the Morphological Matrix Applied to Aircraft Conceptual Design.” Computer-Aided Design 41 (3):187-96. https://doi.org/10.1016/j.cad.2008.06.005. 